\title{
Clearance of amyloid $\beta$-peptide from brain: transport or metabolism?
}

To the editor-We read with interest the article by Iwata et al. ${ }^{1}$ in the February 2000 issue of Nature M edicine suggesting a major degrading catabolic pathway for Alzheimer amyloid $\beta$-peptide $(A \beta)$ in brain. Our own work, however, has indicated that, in addition to production of the peptide, transport of $A \beta$ across the microvascular endothelium (that is, the site of the blood-brain barrier) is essential in controlling $A \beta$ levels in the brain $^{2,3}$.

The physiological relevance of degradation mechanisms of $A \beta$ described by I wata et al. ${ }^{1}$ remains unclear, as the peptide was studied at very high concentrations of about $240 \mu \mathrm{m} / \mathrm{l}$, which may impair blood-brain barrier integrity ${ }^{4,5}$. It is likely that $A \beta$ at a concentration of 240 $\mu \mathrm{m} / \mathrm{l}$ will cause plasma leakage into brain tissue. The size of the spot of Evans blue dye in the hippocampus and along the needle track shown by I wata et al. (ref. 1, Fig. 1d) may translate into a volume of about 20-30 $\mu \mathrm{l}$, much larger than the reported infusion volume of $0.5 \mu \mathrm{l}$. The invasive nature of the acute placement of 26-gauge needle used by I wata et al . ${ }^{1}$ very likely damages the blood-brain barrier. There is injury to blood vessels and microbleeding. Also, staining along the needle track indicates substantial reflux of injected material back to subarchnoid space, where contamination with cere brospinal fluid is obvious. It is essential to place a small 33-gauge guiding cannula at least 3-5 days before infusing peptides to allow the tissue to recover from the trauma.

Finally, the affinity of neprilysin for its physiological substrates (such as enkephalins, tachykinins and atrial natriuretic peptide) and/or different synthetic peptides is in the low millimolar to micromolar range ${ }^{6}$. Normal levels of $A \beta$ in the brain are in the low nanomolar ranges. Even in transgenic models of brain amyloidosis, brain levels of $A \beta$ range from $40 \mathrm{~nm} / \mathrm{kg}$ to $250 \mathrm{~nm} / \mathrm{kg}$ from 3 to 12 months $^{7}$. Several cell surface re ceptors in brain endothelial cells, microglia and/or neurons, including the receptor specific for advanced glycosylation end-products (RAGE), the scavenger type A receptor (SR-A; refs. 2,8), LRP-1 (low density lipoprotein receptor related protein) (ref. 9) and LRP-2 (ref. 3) bind $A \beta$ with high nanomolar affinity directly (RAGE and SR-A) or indirectly (LRP-1 and LRP-2) through ligands such as $\alpha 2 \mathrm{M}$ and apolipoproteins $\mathrm{E}$ and J, which act as high-affinity transport binding proteins for $A \beta$. Thus, in the intact brain, $A \beta$ probably binds to several high-affinity cell surface receptors and/or binding proteins before being recognized by potential degrading enzymes.

\section{BERISLAV V. ZLOKOVIC ${ }^{1}$, SHINYA YAMADA $^{2}$, DAVID HOLTZMAN ${ }^{3}$, JORGE GHISO ${ }^{4} \&$ BLAS FrangIONE ${ }^{4}$ ${ }^{1}$ Center for Aging and Developmental Biology 601 Elmwood Ave., Box 645 \\ Rochester, N ew York, New York 14642 USA Email: berislav_zlokovic@urmc.rochester.edu ${ }^{2}$ Department of Neurosurgery \\ School of M edicine, Tokai University \\ 6-4-14 Ikegami, Tokyo 146, Japan \\ ${ }^{3}$ Department of Neurology \\ Washington University School of M edicine \\ St. Louis, M issouri, 63110 USA \\ ${ }^{4}$ Department of Pathology \\ New York University \\ New York, New York, 10016 USA}

Saido et al. reply-We thank Zlokovic et al. for stimulating this speculation about the functions of transport and catabolism in $A \beta$ clearance from brain. Injection of ${ }^{125}$-labeled $A \beta$ peptides into the lateral ventricle of mouse brain has demonstrated that " $60 \%$ of ${ }^{125} \mathrm{I}-\mathrm{A} \beta_{1-40}$ cleared from the brain in the first 10 min, while only $20 \%$ of ${ }^{125} \mathrm{I}-\mathrm{A} \beta_{1-42}$ was cleared from the brain after 45 min." 10 This is consistent with the sel ective accumulation of $A \beta_{42}$, but not $A \beta_{40}$, in rat brain induced by neutral endopeptidase inhibitor infusion ${ }^{1}$. An appropriate interpretation for these results would be that $A \beta_{40}$ and $A \beta_{42}$ are cleared from brain through distinct mechanisms, and that clearance of $A \beta_{40}$ may be transport-dependent whereas that of $A \beta_{42}$ is more catabolism-dependent, as we discussed ${ }^{1}$.

Indeed, both of the reports that Zlokovic et al. cite to demonstrate that transport of $A \beta$ across the blood-brain barrier is essential in controlling $A \beta$ levels used only $A \beta_{1-40}$ (refs. 2,3). Since the initial reports ${ }^{11}$, the differences between $A \beta_{40}$ and $A \beta_{42}$ in physicochemical, bio- chemical and pathogenic properties have been well established. Because an increase in the ratio of $A \beta_{42}$ to $A \beta_{40}$ is the most commonly accepted phenotype of early onset familial Alzheimer disease-associated gene mutation $\mathrm{s}^{12-14}$, the main focus should be on $A \beta_{42}$ rather than $A \beta_{40}$.

The above arguments, however, do not exclude the possible involvement of a transport system in $A \beta_{42}$ clearance as a secondary or alternative mechanism. It is misleading, however, to claim that "normal levels of $A \beta$ in the brain are in the low nanomolar ranges," because the majority of $A \beta$ is present in the insoluble fractions ${ }^{1}$ where the classical biochemical theory of enzyme kinetics for liquid phase can not be directly applied.

We do agree that the surgical stresses caused by the injection protocols used by us and by Zlokovic et al. could cause artificial effects. For example, placement of a needle, whether 26- or 33-gauge, in brain tissue induces long-term astrocyte activation after a day or two and seems to interfere with metabolism and accumulation of $A \beta$ near the injection site. Therefore, "to place a small 33-gauge guiding cannula at least 3-5 days before infusing" is not necessarily recommended. In our $A \beta$ injection experiments, contamination of the injection area by plasma or cerebrospinal fluid was minimal, as immunohistochemically confirmed using antibody against rat immunoglobulin $\mathrm{G}$, because the needle was kept inserted until immediately before dissection. A sphere of $0.5 \mu \mathrm{l}$ has a radius of $500 \mu \mathrm{m}$. Although efforts to minimize such surgical stresses should be continued, we believe that use of non-invasive techniques, such as 'reverse genetics', will provide a more definite answer in the near future.

Nobuhisa I WATA, SATOSHI TSUBUkI, Emi Hama, Yoshie Takaki, Keiro ShIROTANI \& TaKaOMI C. SaIdo Laboratory for Proteolytic N euroscience RIKEN Brain Science Institute,

Wako-shi 351-0198 JAPAN

Email: saido@brain.riken.go.jp

1. Iwata, $N$. et al. Identification of the major $A \beta_{1-42}$-degrading catabolic pathway in brain parenchyma: Suppression leads to biochemical and pathological deposition. Nature Med. 6, 143-150 (2000). 